\title{
Socio-Demographic Variability in Adolescent Substance Use: Mediation by Parents and Peers
}

\author{
Jing Wang, Bruce G. Simons-Morton, Tilda Farhart, and Jeremy W. Luk \\ Prevention Research Branch, Division of Epidemiology, Statistics, and Prevention Research, \\ Eunice Kennedy Shriver National Institute of Child Health and Human Development, 6100 \\ Building Room 7B13 MSC 7510, Bethesda, MD 20892-7510, USA
}

Jing Wang: wangji2@mail.nih.gov

\begin{abstract}
The current study examined socio-demographic variability in adolescent substance use and the mediating roles of maternal knowledge, paternal knowledge and peer substance use. The data were obtained from the United States records $(N=8,795)$ of the Health Behavior in School-Aged Children 2005/2006 Survey, in grades 6 through 10. The analyses employed multiple indicator multiple cause and structural equation models. Adolescent substance use was measured by frequencies of alcohol use, being drunk, and cigarette and marijuana use in the past month. Peer influence had a direct influence on adolescent substance use. Maternal knowledge had both direct and indirect influences on adolescent substance use through its negative association with substance-using peers, whereas paternal knowledge only had an indirect influence. Parental knowledge and peer substance use totally mediated differences in adolescent substance use by grade; differences between Caucasian and African-American or Hispanic adolescents; and differences between adolescents from two-parent families and those from single-mother, singlefather or mother-stepfather families. Parental knowledge and peer substance were important mediators which largely accounted for variability in the prevalence of adolescent substance use by grade, race/ethnicity, and family structure.
\end{abstract}

\section{Keywords}

Adolescent substance use; Parental knowledge; Peer substance use; Socio-demographic differences; Family structure

\begin{abstract}
Adolescent substance use is often linked to socio-demographic variables such as gender, age, race/ethnicity and family structure (Blum et al. 2000). Substantial literature indicates that elevated risks for substance use are found among older adolescents, Caucasians, and those who were raised in a single-parent family (Barrett and Turner 2006; Johnston et al. 2007; Kuntsche and Silbereisen 2004). Traditionally, the prevalence of substance use is higher among male than female adolescents, although emerging evidence suggests that this pattern has changed in the past few decades (Hammond 2009; Keyes et al. 2008). Despite our knowledge regarding socio-demographic variability in adolescent substance use, it remains unclear how our knowledge can be best translated into prevention efforts because socio-demographic variables are generally not modifiable. However, it may be possible to design interventions that address specific demographic groups, to the extent that modifiable factors associated with substance use in demographic subgroups can be identified. The
\end{abstract}

C) Society for Prevention Research 2009

Correspondence to: Jing Wang, wang ji2@mail.nih.gov. 
present study extends previous investigations on socio-demographic variability in adolescent substance use by examining the mediating roles of paternal knowledge, maternal knowledge, and peer substance use, three important and modifiable social influences on adolescent substance use.

\section{Social Influences on Substance Use}

Effective parenting is inversely associated with adolescent substance use (DeVore and Ginsburg 2005). Among various parenting practices, parental knowledge is an important construct that reflects reasonable parent-child communication and relations leading to parental awareness of their adolescents' friends, activities, and whereabouts (Hayes et al. 2003; Kerr and Stattin 2000; Stattin and Kerr 2000). Previous studies have shown that parental knowledge is a protective factor against adolescent use of cigarettes (SimonsMorton et al. 2001), alcohol (Barnes and Farrell 1992), and marijuana (Hoffmann 2002), although there is disagreement as to whether parental knowledge is associated with adolescent substance use directly or indirectly through other variables such as peer substance use.

Steinberg et al. (1994), for instance, found that parental knowledge has a direct protective effect but not an indirect effect on adolescents' use of alcohol, marijuana and other substances in a sample of adolescents in Wisconsin and northern California. Two other studies conducted in Maryland, however, showed that parental knowledge has both direct and indirect protective effects on adolescent smoking and drinking progression, and the indirect effect was through substance-using friends (Simons-Morton 2007; Simons-Morton et al. 2004). Given the few studies on this topic, it is not yet clear the extent to which parental knowledge provides direct or indirect protective effects on adolescent substance use. It is possible that inconsistencies in the current findings are due to the use of different samples, characterized by different age groups, race/ethnic composition, and urban or rural locations, highlighting the need to test the relationships between parental knowledge, peer substance use and adolescent substance use in a diverse and nationally representative sample.

Another gap in the parental knowledge and substance use literature is that few studies have examined paternal and maternal knowledge separately. Mixed findings were reported among the few studies that did (e.g., Waizenhofer et al. 2004). For example, Coley et al. (2008) reported that knowledge from either parent was protective against adolescent substance use with a similar magnitude. However, another study found that paternal knowledge was not a significant factor when maternal knowledge was controlled for (Waizenhofer et al. 2004). Based on research showing that fathers are often less involved in parenting than mothers (Williams and Kelly 2005), it is of interest to examine whether maternal knowledge may have a stronger association with adolescent substance use than paternal knowledge.

Contrary to the mixed findings in the parental knowledge literature, empirical studies on peer influence have consistently shown that peer substance use is among the strongest correlates or predictors of adolescent substance use (Hawkins et al. 1992; Hoffman et al. 2007). Concurrent and prospective analyses indicate that adolescents who associate with substance-using peers are more likely to smoke cigarettes (Simons-Morton 2002), drink alcohol (Simons-Morton et al. 2004), use marijuana (Bahr et al. 2005), or use other illicit drugs (Stanton et al. 2002). These findings are consistent with the problem behavior theory, which posits that associating with deviant peers increases the adoption of similar problem behaviors (Jessor and Jessor 1977). Findings from the peer substance use and parental knowledge literature suggest that both are linked to adolescent substance use, but that peer 
substance use is a stronger correlate that may mediate the association between parental knowledge and adolescent substance use (e.g., Simons-Morton 2007).

\section{Parental and Peer Influences as Mediators}

Given the importance of parental knowledge and peer substance use in adolescent substance use prevention, it is of interest to examine whether these modifiable variables mediate the association between adolescent substance use and socio-demographic characteristics, including gender, age, race/ethnicity and family structure. Evidence suggests that, relative to boys and older adolescents, girls and younger adolescents experience a higher level of parental knowledge, which may in turn protect them from using substances (Barnes et al. 2000). Similarly, a recent study on racial/ethnic differences in alcohol use demonstrated that African-Americans are less influenced by their friends who drank alcohol but are more influenced by parental support than Caucasians, which may partially explain the corresponding differences in adolescent alcohol use (Watt and Rogers 2007). Likewise, parental and peer relations may mediate the association between family structure and adolescent substance use. Adolescents from intact families are less likely to use substances than those from non-intact families (Hoffmann 2002). Previous studies have also examined parenting practices and peer affiliations as potential mediators of the family structuresubstance use relationship. For example, Broman et al. (2008) found that after controlling for parenting, peer use, religiosity and neighborhood problems, the direct effect of family structure on adolescent substance use was minimal. These findings suggest that gender, age, racial/ethnic and family structure differences in adolescent substance use may be mediated by parental and peer variables such as parental knowledge and peer substance use.

\section{The Scope of the Present Study}

The purpose of the current study was to examine the socio-demographic variability in adolescent substance use and the mediating roles of paternal knowledge, maternal knowledge, and peer substance use in a diverse and nationally representative sample. The following research questions were addressed: (a) What is the variability in adolescent substance use by gender, grade, race/ethnicity, and family structure? (b) Are there both direct and indirect influences of maternal knowledge and paternal knowledge on adolescent substance use? and (c) To what extent can maternal knowledge, paternal knowledge and peer substance use mediate the socio-demographic variability in adolescent substance use?

\section{Method}

\section{Sample and Procedure}

The data were obtained from the United States records $(N=9011)$ of the Health Behavior in School-Aged Children (HBSC) 2005/2006 Survey, a World Health Organization collaborative cross-national study examining health behaviors among children and their social determinants. A three-stage stratified sampling design was implemented for the selection of students in grades 6 through 10 in the United States. School districts, schools and then school classes were selected for study using a weighted probability technique to produce a nationally representative sample of students with an oversample of AfricanAmerican and Hispanic students. This sampling design was fully taken into account in the analysis, through the special features dealing with complex survey data in Mplus, version 5 (Muthén and Muthén 2008), the software used for the current study. Data were collected through anonymous self-report questionnaires distributed in the classroom, with a student response rate of $85 \%$. Youth assent and parental consent were obtained as required by the participating school districts. The study protocol was reviewed and approved by the 
Institutional Review Board of the Eunice Kennedy Shriver National Institute of Child Health and Human Development.

\section{Measures}

Socio-Demographic Characteristics-Gender was measured as male or female. Grade was included as a continuous variable with five levels: 6 to 10. Race/ethnicity was measured as a four-category variable: Caucasian, African-American, Hispanic, and other races or ethnicities. Family structure was measured with six categories: two-parent, single-mother, single-father, mother-stepfather, father-stepmother, and others. SES was used as a control variable in the current study as it is related to race/ethnicity and family structure. To avoid too many missing values on the traditional measures of SES, such as family income or parental education, we used the family affluence scale, FAS, developed especially for the HBSC, as the proxy for SES (Currie et al. 2008). It consists of four items assessing family material wealth (i.e., having own bedroom, number of times on a traveling vacation in a year, number of home computers and number of cars owned). This scale has shown desirable reliability and validity (Schnohr et al. 2007). The four items were combined to produce a linear composite score, with a range from 0 (lowest affluence) to 9 (highest affluence).

Outcome Variable and Mediators-The outcome variable (substance use) and the three mediators (maternal knowledge, paternal knowledge and peer substance use) were all latent variables or factors, each measured by several items. In preliminary analyses, a series of confirmatory factor analyses were conducted to test the factor structures for each of the four factors. The results showed desirable goodness-of-fits and confirmed the measurement models.

Adolescent substance use was measured by asking students the number of occasions in the last 30 days they had (1) drunk alcohol; (2) been drunk; (3) smoked cigarettes; and (4) taken marijuana. The five-point scale ranged from 0 (never) to 4 (more than 10 times). Peer substance use was assessed by four questions that asked respondents how many of their friends (1) drank alcohol; (2) had been drunk at least once every week; (3) smoked cigarettes; and (4) used marijuana. The scale ranged from 0 (none) to 4 (all). Maternal and paternal knowledge were measured separately by adolescents' perceived maternal (or female guardian's) and paternal (or male guardian's) awareness of the following items: (1) who their friends were; (2) how they spent their money; (3) where they were after school; (4) where they went at night; and (5) what they did with their free time. Both maternal knowledge and paternal knowledge had four-point scale responses. For instance, response choices for maternal knowledge were 0 (Don't have/see mother/guardian), 1 (She doesn't know anything), 2 (she knows a little) and 3 (she knows a lot).

\section{Analysis}

Our analyses consisted of two steps. The first step was to develop a multiple indicator multiple cause model, MIMIC model, with the purpose of examining the variability in adolescent substance use by socio-demographic variables, including gender, grade, race/ ethnicity, family structure and FAS. The second step was to further generate a structural equation model, SEM, by adding the three hypothesized mediators, maternal knowledge, paternal knowledge and substance-using peers, as shown in Fig. 1. The model tested four regressions simultaneously: (1) a regression of maternal knowledge on socio-demographic variables; (2) a regression of paternal knowledge on socio-demographic variables; (3) a regression of substance-using peers on maternal knowledge, paternal knowledge, and sociodemographic variables; and (4) a regression of adolescent substance use on maternal knowledge, paternal knowledge, substance-using peers, and socio-demographic variables. 
As shown in Fig. 1, the SEM allows us to test four sets of pathways leading to adolescent substance use: (1) pathway from substance-using peers to adolescent substance use showing peer influence; (2) pathways from maternal or paternal knowledge to adolescent substance use, showing their direct influences on adolescent substance use; (3) pathways from maternal or paternal knowledge to substance-using peers, and then to adolescent substance use, showing their indirect influences on adolescent substance use through substance-using peers; and (4) pathways from each of the socio-demographic variables to maternal knowledge, paternal knowledge, substance-using peers, and adolescent substance use, testing the socio-demographic differences in the outcome variable and in the three mediators simultaneously. We also expected maternal knowledge and paternal knowledge to be positively correlated. In addition, as shown in Fig. 1, we have the same measures for maternal knowledge and paternal knowledge, and one-to-one corresponding substance use items for adolescents and their peers, so we defined the measurement errors for the paired items to be correlated.

A mediating effect is evident when there are significant paths between adolescent substance use and the three mediators, between adolescent substance use and the socio-demographic variables, and between the mediators and the socio-demographic variables (Baron and Kenny 1986). A significant path from a socio-demographic variable to adolescent substance use in the MIMIC model that becomes non-significant in the SEM would indicate a total mediation. Significance level was set as 0.05 .

All models were tested with the software package Mplus (Muthén and Muthén 2008). Mplus allows the use of both continuous and ordinal indicators as independent and dependent variables. In our models, we had a mixture of both types of variables. For example, the variables of adolescent substance use, peer substance use, maternal and paternal knowledge were all strongly skewed and were configured as ordinal variables. Another advantage of using Mplus is that it enabled us to make use of all available data, including cases with some missing responses, through the estimation of Full Information Maximum Likelihood (FIML) (Schafer and Graham 2002). To evaluate the fit of the models, we used three goodness-of-fit indexes, CFI, TLI, and RMSEA (Kline 1998). Usually CFI and TLI values above 0.90 and RMSEA less than or equal to 0.06 indicate acceptable fit (Hu and Bentler 1999). Chi-square statistics were less suitable in our study, given the large sample size.

\section{Results}

\section{Sample Characteristics}

Of the 9,011 adolescents who completed the HBSC survey of 2005, 216 (2.4\%) were excluded from our analyses due to missing information on the socio-demographic variables. Sample characteristics for the remaining 8,795 adolescents are reported in Table 1.

\section{Prevalence of Adolescent Substance Use}

We report in Table 1 the percentages of adolescents who had experience with drinking alcohol, being drunk, smoking cigarettes, or using marijuana for at least once in the last 30 days. About $32 \%$ of adolescents had drunk alcohol and $18.1 \%$ had been drunk in the past 30 days, $13.6 \%$ had smoked cigarettes, and $8.9 \%$ had used marijuana.

\section{Socio-Demographic Variability in Substance Use}

The MIMIC model tested the associations between the latent variable of adolescent substance use and the four socio-demographic variables; i.e., gender, grade, race/ethnicity, and family structure, with FAS as the control variable. The results showed a desirable 
goodness-of-fit, $\mathrm{CFI}=0.991$, TLI $=0.990$, and RMSEA $=0.027$. The regression coefficients in the MIMIC model are listed in the second column of Table 2.

As shown in Table 2, there was no significant gender difference in adolescent substance use, with controlling for other socio-demographic variables. Higher grade was associated with more substance use (the unstandardized regression coefficient, $\beta=0.235, p<0.001$ ). With Caucasian adolescents as the referent, African-American adolescents reported less substance use and Hispanic adolescents reported more use. With adolescents from two-parent families as the referent, those from all five non-intact families reported more substance use. Family affluence had a positive relation with substance use, but the magnitude of the association was small (the standardized regression coefficient, $\beta=0.019$ ).

\section{Direct and Indirect Influences of Maternal and Paternal Knowledge}

The direct and indirect influences of maternal and paternal knowledge were tested in the SEM, shown in the third through six columns in Table 2 under Model 2. Indices of goodness-of-fit of the SEM showed a desirable fit, CFI=0.986, TLI=0.986, and RMSEA=0.029. The SEM tested four regressions simultaneously, involving both observed and latent variables. The regression coefficients are reported in columns three to six of Table 2 .

Direct Influences-The last two columns in Table 2 contain coefficients for the two regressions with substance-using peers and adolescent substance use as outcome variables. As shown in the last column, peer substance use was strongly associated with adolescent substance use $(\beta=0.629)$. Maternal knowledge was negatively associated with adolescent substance use, $(\beta=-0.195)$, illustrating a direct influence. The direct influence of paternal knowledge on substance use was -0.046 , and was only marginally significant $(0.05<p<0.10)$.

Indirect Influences-Maternal and paternal knowledge would have indirect influences on adolescent substance use if they are associated with peer substance use. As shown in column five of Table 2, maternal and paternal knowledge were both negatively associated with substance-using peers ( $\beta=-0.338$ for maternal knowledge and -0.099 for paternal knowledge). Thus, both maternal knowledge and paternal knowledge had indirect influences on adolescent substance use, with the standardized magnitudes of 0.213 , which was the product of 0.629 and 0.338 , for maternal knowledge, and 0.062 for paternal knowledge.

\section{Mediating Roles of Parental Knowledge and Peer Substance Use}

By comparing the regression coefficient of each socio-demographic variable on adolescent substance use in the MIMIC model with the corresponding coefficient in the SEM, we can test the mediating roles of maternal knowledge, paternal knowledge and peer substance use.

Gender-As shown in Table 2, there was no gender difference in substance use in either the MIMIC model or in the SEM.

Grade-Grade difference in substance use was significant in the MIMIC model, but not significant in the SEM, indicating a total mediation. By examining grade differences in the three mediators, we found that older adolescents reported less maternal knowledge, less paternal knowledge and more substance-using peers.

Race/Ethnicity-The racial/ethnic differences in substance use between Caucasian and African-American adolescents or between Caucasian and Hispanic adolescents, which were significant in the MIMIC model, became non-significant in the SEM, indicating a total 
mediation. Racial/ethnic differences were found in all three mediators. Specifically, AfricanAmerican adolescents reported less maternal knowledge, less paternal knowledge, but also fewer substance-using peers. Hispanic adolescents reported less maternal and paternal knowledge. The racial/ethnic differences in maternal knowledge, paternal knowledge and substance-using peers totally explained the racial/ethnic differences in adolescent substance use.

Family Structure-With two-parent family structure as the referent, the adolescent substance use difference between two-parent family structure and father-stepmother or other family structure was still significant, but there was no difference between adolescents from two-parent and those from mother-only, father-only, or mother-stepfather family structures. By examining the family structure differences in the three mediators, we found that adolescents from all five non-intact families reported less maternal knowledge, especially for those from the two father-custody families (single-father: $\beta=-0.578$ and fatherstepmother: $\beta=-0.292$ ). Furthermore, less paternal knowledge was observed among adolescents in single-mother, mother-stepfather or other family structures, whereas more paternal knowledge was found among those from father-only families. In addition, those from the two step-parents families reported more substance-using friends (motherstepfather: $\beta=0.121$ and father-stepmother: $\beta=0.176$ ).

Family Affluence-Family affluence had a significant influence on substance use in both the MIMIC model and the SEM, which showed no clear signs of mediation. However, it is interesting to note that a higher level of family affluence was not only related to more maternal knowledge, more paternal knowledge, but also more substance-using peers.

\section{Discussion}

The purpose of the current study was to examine the socio-demographic variability in adolescent substance use and the mediating roles of parental knowledge and peer substance use. We found that maternal knowledge had both direct and indirect influences on adolescent substance use, whereas paternal knowledge only had an indirect effect. Maternal knowledge, paternal knowledge and peer substance use totally mediated differences in adolescent substance use by grade, differences between Caucasian adolescents and AfricanAmerican or Hispanic adolescents, differences between adolescents from two-parent families and those from single-mother, single-father, or mother-stepfather families.

\section{Direct and Indirect Influences of Maternal and Paternal Knowledge}

Our results on the direct and indirect influences of maternal knowledge and the indirect influence of paternal knowledge are consistent with its protective influence on adolescent substance use both directly and indirectly through buffering adolescents' association with substance-using peers found in previous studies (Simons-Morton 2007; Simons-Morton et al. 2004). Maternal knowledge had both direct and indirect influences, with similar magnitudes. Paternal knowledge was positively correlated with maternal knowledge, but it had only a marginally significant direct influence when parallel with maternal knowledge. This is consistent with findings of Waizenhofer and colleagues' (2004) study. Waizenhofer et al. examined the methods mothers and fathers obtained their information used to obtain their knowledge about their children. They found that mothers mainly obtained their knowledge through children's disclosure of information, whereas fathers obtained their information mainly through their communications with mothers. Similarly, a series of studies by Stattin and Kerr showed that parental knowledge was gained mainly through child disclosure of information, and not by monitoring through tracking and overt surveillance (Kerr and Stattin 2000; Stattin and Kerr 2000). They suggested it could be the emotional 
attachment between adolescents and their parents that caused adolescents to communicate with their parents and to be better adjusted. In addition, the indirect influence of paternal knowledge indicates that fathers may have protective influences on adolescent substance use by reducing the likelihood of forming friendships with deviant peers, and this protective influence is independent from maternal influences.

\section{Mediating Roles of Parental Knowledge and Peer Substance Use}

Gender-We did not find any gender differences in adolescent substance use in either the MIMIC model or SEM. However, gender was related to all three mediators, in that girls reported more maternal knowledge, less paternal knowledge and more substance-using peers. The gender difference in maternal and paternal knowledge is consistent with literature that parents have more knowledge about their same-sex children (Crouter et al. 1999). The relation between gender and substance-using peers is analogous to previous studies that girls are more susceptible to peer influence (e.g., Simons-Morton et al. 2001). However, our study shows that boys and girls differ on the numbers of substance-using peers, rather than the strength of the association between adolescent and peer substance use.

Grade-As there was no grade difference in adolescent substance use in the SEM, we can conclude that parental knowledge and peer substance use totally mediated grade variability in adolescent substance use. The total mediation of grade differences shows the important roles played by parental knowledge and peer substance use, especially from a developmental perspective. We found that older adolescents reported less maternal knowledge, less paternal knowledge, and more substance-using peers, which was consistent with previous studies (Barnes et al. 2000; Simons-Morton et al. 2001).

Race/Ethnicity-Parental knowledge and substance-using peers also mediated racial/ ethnic variability in adolescent substance use. Specifically, African-American adolescents reported less maternal knowledge, less paternal knowledge, and fewer substance-using friends, which collectively explained the differences in substance use between them and Caucasian adolescents. Hispanic adolescents reported less maternal and paternal knowledge, which in turn explained their higher frequency of substance use compared to Caucasian adolescents.

Family Structure-All five categories of non-intact families were related to higher frequencies of substance use, a finding consistent with previous studies (e.g., Hoffmann 2002). When linking family structure to the three mediators, we found that adolescents in all five non-intact families reported less maternal knowledge, especially for those in father-only or father-stepmother families. Adolescents in the three father-absent families; i.e., motheronly, mother-stepfather and other family structure, reported less paternal knowledge, whereas those in father-only families reported less paternal knowledge. We also found more substance-using peers among adolescents in the mother-stepfather and father-stepmother families. Less maternal knowledge, less paternal knowledge, and more substance-using peers totally mediated family structure differences in adolescents from the two-parent families and those from single-mother, single-father or mother-stepfather families.

\section{Contributions to the Literature}

This research can contribute to the literature in at least three ways. First, we studied the separate roles of mothers and fathers on adolescent substance use. By separating paternal knowledge from maternal knowledge, father-only families from mother-only families, and father-stepmother families from mother-stepfather families, we uncovered different associations between these variables and adolescent substance use. For example, we found that maternal knowledge had both direct and indirect influences on substance use, whereas 
paternal knowledge had only an indirect influence through its negative association with substance-using peers.

Second, a large representative US sample, with over-sampling of African-American and Hispanic adolescents, allowed us to examine the differential contribution of race/ethnicity, SES, and family structure on adolescent substance use. Previous studies showed mixed findings when examining racial/ethnic, SES or family structure differences in adolescent substance use (Hoffmann 2002). A possible explanation for the conflicting results may be due to the inter-correlation between race/ethnicity, SES and family structure. For example, African-American adolescents are found more likely to come from poor and single-parent families (Blum et al. 2000). Another explanation relates to the use of less representative samples, or insufficient numbers of certain racial/ethnic groups in the samples. For instance, substance use among Hispanic adolescents has been found to vary widely across studies, suggesting that larger and more representative samples are needed to detect the difference between Caucasian and Hispanic adolescents (Blum et al. 2000; Khoury et al. 1996). In addition, the nationally representative sample in the current study allowed us to generalize the results to the population of US adolescents.

Third, the latent variable approach and the structural equation modeling we used in the current study have several advantages. For example, the estimated associations between the latent variables are unattenuated by modeling measurement errors. We also tested several regressions simultaneously without inflating type I errors. In addition, the flexibility of structural equation modeling allows us to include the correlation of measurement errors between the same substance used by adolescents and their peers. We found that the correlation was 0.163 for drinking alcohol, 0.059 for being drunk, 0.061 for smoking cigarettes, and 0.230 for smoking marijuana. It is consistent with the notion that the peer use of a particular substance has higher influence on adolescent use of the same substance. Moreover, this is also consistent with studies suggesting greater influence of marijuanausing peers on adolescent use of marijuana (Bahr et al. 2005; Kandel 1980). Thus, even though we used the latent variables for adolescent substance use and substance-using peers, showing the overall levels of alcohol, cigarette, and marijuana use, we also considered that a particular substance used by peers may have extra influence on adolescent use of the same item.

\section{Limitations and Implications}

It is also important to note the limitations of this study. First, while we have used the term "influence" to describe relationships between peer and adolescent substance use, the crosssectional nature of the study limits conclusions about causal associations between adolescent and peer substance use. For instance, some researchers suggest both peer socialization and peer selection contribute to the initiation and progression of adolescent substance use (Simons-Morton 2007), a topic that was beyond the scope of the study. Longitudinal studies are needed to further examine both pathways of peer socialization and peer selection. Second, all the information was reported by the adolescent participants. Self-reported substance use is generally considered reliable (Post et al. 2005; Campanelli et al. 1987), but self-reports on substance-using peers may be inflated for those who currently use substances (Bauman and Ennett 1998). Third, the SEM in our analyses assumes no moderating effects of any socio-demographic variable on the association between parental knowledge, peer substance use and adolescent substance use. Even though the model shows desirable goodness-of-fit, the variation of the association between parental knowledge, peer substance use and adolescent substance use by socio-demographic characteristics cannot be ruled out. Finally, the current study used a quantitative approach to examine the association between parental knowledge and substance use of adolescents and their peers. Future in-depth 
qualitative studies are recommended to identify new theoretical aspects of how parental knowledge is actualized in real life.

Nonetheless, the study findings indicate that maternal knowledge, paternal knowledge and peer substance use contribute to the explanation of the variability in adolescent substance use by grade, race/ethnicity and family structure. The information on socio-demographic differences in adolescent substance use, as well as their differences in parental knowledge and substance-using peers, should be useful for guiding prospective and intervention studies. Specific implications of the current study for future research, prevention practices and program development include: (1) additional research is needed to determine how peer substance use mediates the relationship between parental knowledge and adolescent substance use; (2) further research is needed to examine the mechanisms by which parent knowledge affects adolescent substance use (i.e., is it the effect of parents' actual knowledge about their adolescent child or does parent knowledge mainly reflect the parent-child relationship?); (3) prevention programs should be designed to increase parental knowledge and moderate peer influence, particularly among adolescent who report lower level of parental knowledge and relatively more substance using peers.

\section{Acknowledgments}

This research was supported by the Intramural Research Program of the Eunice Kennedy Shriver National Institute of Child Health and Human Development and the Maternal and Child Health Bureau of the Health Resources and Services Administration.

\section{References}

Bahr SJ, Hoffman KP, Yang X. Parental and peer influences on the risk of adolescent drug use. Journal of Primary Prevention. 2005; 26:529-551. [PubMed: 16228115]

Barnes GM, Farrell MP. Parental support and control as predictors of adolescent drinking, delinquency, and related problem behaviors. Journal of Marriage and the Family. 1992; 54:763776.

Barnes GM, Reifman AS, Farrell MP, Dintcheff BA. The effects of parenting on the development of adolescent alcohol misuse: A six-wave latent growth model. Journal of Marriage and Family. 2000; 62:175-186.

Baron RM, Kenny DA. The moderator-mediator variable distinction in social psychological research: Conceptual, strategic, and statistical considerations. Journal of Personality and Social Psychology. 1986; 51:1173-1182. [PubMed: 3806354]

Barrett AE, Turner J. Family structure and substance use problems in adolescence and early adulthood: Examining explanations for the relationship. Addiction. 2006; 101:109-120. [PubMed: 16393197]

Bauman KB, Ennett ST. Validity of adolescent self-reports of cigarette smoking. American Journal of Public Health. 1998; 88:309-310. [PubMed: 9491034]

Blum RW, Beuhring T, Shew ML, Bearinger LH, Sieving RE, Resnick MD. The effects of race/ ethnicity, income, and family structure on adolescent risk behaviors. American Journal of Public Health. 2000; 90:1879-1884. [PubMed: 11111260]

Broman CL, Li X, Reckase M. Family structure and mediators of adolescent drug use. Journal of Family Issues. 2008; 29:1625-1649.

Campanelli PC, Dielman TE, Shope JT. Validity of adolescents' self-reports of alcohol use and misuse using a bogus pipeline procedure. Adolescence. 1987; 22:7-22. [PubMed: 3591505]

Coley RL, Votruba-Drzal E, Schindler HS. Trajectories of parenting processes and adolescent substance use: Reciprocal effects. Journal of Abnormal Child Psychology. 2008; 36:613-625. [PubMed: 18288605]

Crouter AC, Helms-Erikson H, Updegraff K, McHale SM. Conditions underlying parents' knowledge about children's daily lives in middle childhood: Between- and within-family comparisons. Child Development. 1999; 70:246-259. [PubMed: 10191526] 
Currie C, Molcho M, Boyce W, Holstein B, Torsheim T, Richter M. Researching health inequalities in adolescents: The development of the Health Behaviour in School-Aged Children (HBSC) Family Affluence Scale. Social Science \& Medicine. 2008; 66:1429-1436. [PubMed: 18179852]

DeVore ER, Ginsburg KR. The protective effects of good parenting on adolescents. Current Opinion in Pediatrics. 2005; 17:460-465. [PubMed: 16012256]

Hammond SK. Global patterns of nicotine and tobacco consumption. Handbook of Experimental Pharmacology. 2009; 192:3-38. [PubMed: 19184644]

Hawkins JD, Catalano RF, Miller JY. Risk and protective factors for alcohol and other drug problems in adolescence and early adulthood: Implications for substance abuse prevention. Psychological Bulletin. 1992; 112:64-105. [PubMed: 1529040]

Hayes LL, Hudson A, Matthews J. Parental monitoring: A process model of parent-adolescent interaction. Behaviour Change. 2003; 20:13-24.

Hoffmann JP. The community context of family structure and adolescent drug use. Journal of Marriage and Family. 2002; 64:314-330.

Hoffman BR, Monge PR, Chou CP, Valente TW. Perceived peer influence and peer selection on adolescent smoking. Addictive Behaviors. 2007; 32:1546-1554. [PubMed: 17188818]

$\mathrm{Hu}$ L, Bentler PM. Cutoff criteria for fit indexes in covariance structure analysis: Conventional criteria versus new alternatives. Structural Equation Modeling. 1999; 6:1-55.

Jessor, R.; Jessor, SL. Problem behavior and psychosocial development: A longitudinal study of youth. New York: Academic; 1977.

Johnston, LD.; O'Malley, PM.; Bachman, JG.; Schulenberg, JE. Monitoring the Future national survey results on drug use, 1975-2006: Volume I, Secondary school students (NIH Publication No. 07-6205). Bethesda, MD: National Institute on Drug Abuse; 2007.

Kandel, DB. Drug and drinking behavior among youth. In: Coleman, J.; Inkeles, A.; Smelser, N., editors. Annual review of sociology. Vol. 6. 1980. p. 235-285.

Kerr M, Stattin H. What parents know, how they know it, and several forms of adolescent adjustment: Further support for a reinterpretation of monitoring. Developmental Psychology. 2000; 36:366380. [PubMed: 10830980]

Keyes KM, Grant BF, Hasin DS. Evidence for a closing gender gap in alcohol use, abuse, and dependence in the United States population. Drug and Alcohol Dependence. 2008; 93:21-29. [PubMed: 17980512]

Khoury EL, Warheit GJ, Zimmerman RS, Vega WA, Gil AG. Gender and ethnic differences in the prevalence of alcohol, cigarette, and illicit drug use over time in a cohort of young Hispanic adolescents in south Florida. Women Health. 1996; 24:21-40. [PubMed: 8883369]

Kline, RB. Principles and practice of structural equation modeling. New York: Guilford; 1998.

Kuntsche EN, Silbereisen RK. Parental closeness and adolescent substance use in single and twoparent families in Switzerland. Swiss Journal of Psychology. 2004; 63:85-92.

Muthén, LK.; Muthén, BO. Mplus user's guide. 5. Los Angeles, CA: Authors; 1998-2008.

Post A, Gilljam H, Rosendahl I, Meurling L, Bremberg S, Galanti MR. Validity of self reports in a cohort of Swedish adolescent smokers and smokeless tobacco (snus) users. Tobacco Control. 2005; 14:114-117. [PubMed: 15791021]

Schafer JL, Graham JW. Missing data: Our view of the state of the art. Psychological Methods. 2002; 7:147-177. [PubMed: 12090408]

Schnohr C, Kreiner S, Due P, Currie C, Boyce W, Diderichsen F. Differential item functioning of a family affluence scale: Validation study on data from HBSC 2001/02. Social Indicators Research. 2007; 89:79-95.

Simons-Morton BG. Prospective analysis of peer and parent influences on smoking initiation among early adolescents. Prevention Science. 2002; 3:275-283. [PubMed: 12465590]

Simons-Morton BG. Social influences on adolescent substance use. American Journal of Health Behavior. 2007; 31:672-684. [PubMed: 17691881]

Simons-Morton B, Haynie DL, Crump AD, Saylor KE. Peer and parent influences on smoking and drinking among early adolescents. Health Education and Behavior. 2001; 28:95-107. [PubMed: 11213145] 
Simons-Morton BG, Chen R, Abroms R, Haynie DL. Latent growth curve analyses of peer and parent influences on smoking progression among early adolescents. Health Psychology. 2004; 23:612621. [PubMed: 15546229]

Stanton B, Li X, Pack R, Cottrell L, Harris C, Burns JM. Longitudinal influence of perceptions of peer and parental factors on African American adolescent risk involvement. Journal of Urban Health. 2002; 79:536-548. [PubMed: 12468673]

Stattin H, Kerr M. Parental monitoring: A reinterpretation. Child Development. 2000; 71:1072-1085. [PubMed: 11016567]

Steinberg L, Fletcher A, Darling N. Parental monitoring and peer influences on adolescent substance use. Pediatrics. 1994; 93:1060-1064. [PubMed: 8197008]

Waizenhofer RN, Buchanan CM, Jackson-Newsom J. Mothers' and fathers' knowledge of adolescents' daily activities: Its sources and its link with adolescent adjustment. Journal of Family Psychology. 2004; 18:348-360. [PubMed: 15222842]

Watt TT, Rogers JM. Factors contributing to differences in substance use among Black and White adolescents. Youth Society. 2007; 39:54-74.

Williams SK, Kelly FD. Relationships among involvement, attachment, and behavioral problems in adolescence: Examining father's influence. Journal of Early Adolescence. 2005; 25:168-196. 


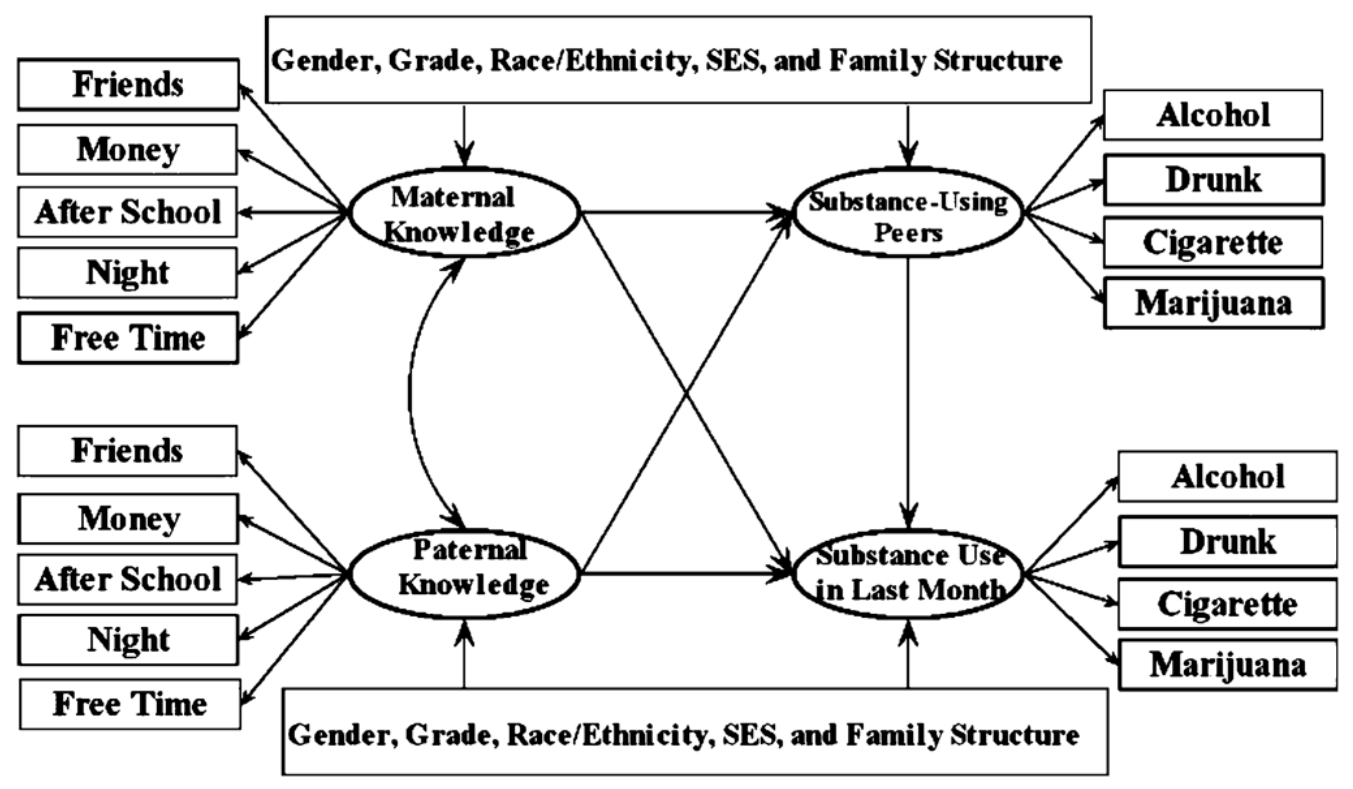

Fig. 1.

Structural equation model: mediating roles of parental knowledge and peer substance use. Maternal knowledge, paternal knowledge, substance-using peers, and adolescent substance use are all treated as latent variables and are placed in ovals. Each of the four latent variables is measured by several ordinal observed variables, which are placed in rectangles. For simplicity, correlations of measurement errors are not shown in the figure. There are two sets of measurement errors. One set is for the correlation between the same measures of knowledge from mothers and fathers. The other set is for the correlation between the same measures of substance use for adolescents and their peers 


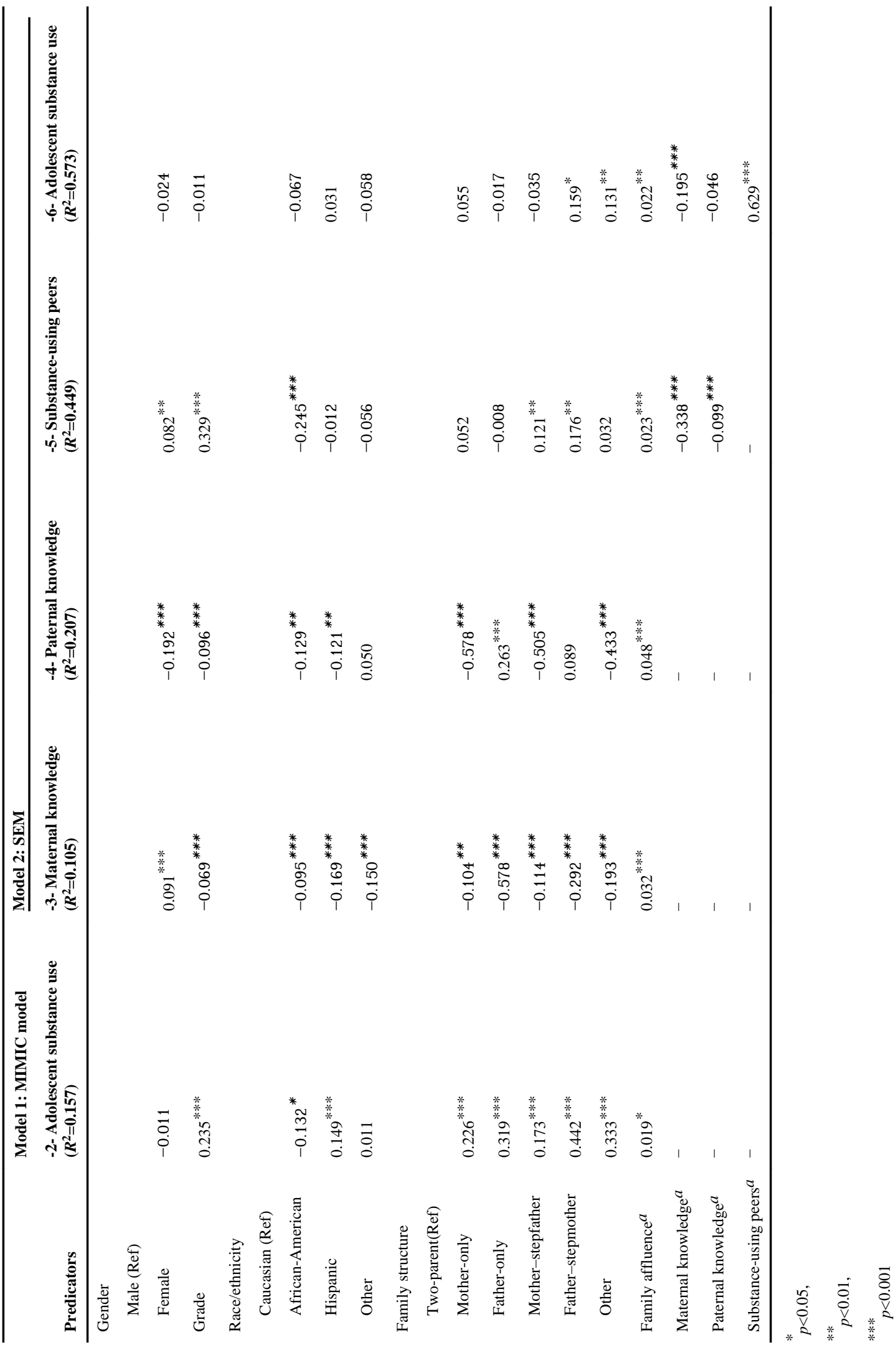


